\title{
MicroRNA-let-7e regulates the progression and development of allergic rhinitis by targeting suppressor of cytokine signaling 4 and activating Janus kinase 1/signal transducer and activator of transcription 3 pathway
}

\author{
LIHUA LI ${ }^{*}$, SHAORONG ZHANG ${ }^{*}$, XUNSHUO JIANG, YUEHUI LIU, KE LIU and CHUNPING YANG \\ Department of Otorhinolaryngology, Head and Neck Surgery, \\ The Second Affiliated Hospital of Nanchang University, Nanchang, Jiangxi 330006, P.R. China
}

Received October 13, 2016; Accepted July 25, 2017

DOI: $10.3892 /$ etm.2018.5827

\begin{abstract}
The present study aimed to explore the microRNA-let-7e (miR-let-7e) expression in allergic rhinitis (AR), and to investigate the underlying molecular mechanisms. miR-let-7e expression in the nasal mucosa of mice and patients with AR were detected. The expression levels of three inflammatory factors, including histamine, immunoglobulin $\mathrm{E}$ and tumor necrosis factor- $\alpha$ (TNF- $\alpha$ ), in the blood of AR mice and in interleukin (IL)-13-stimulated nasal epithelial cells (NECs) were also measured. Furthermore, the target gene of miR-let-7e was predicted and validated using a luciferase reporter assay. The expression levels of Janus kinase 1 (JAK1) and signal transducer and activator of transcription 3 (STAT3) were detected. The results demonstrated that miR-let-7e was downregulated in patients and mice with AR compared with the controls. In addition, the expression levels of inflammatory factors were higher in the blood of mice with AR compared with the control group, while miR-let-7e overexpression inhibited these levels in AR mice and IL-13-stimulated NECs. Furthermore, suppressor of cytokine signaling 4 (SOCS4) was revealed as a potential target gene of miR-let-7e and was negatively regulated by miR-let-7e. Overexpression of SOCS4 abrogated the anti-inflammatory activity of miR-let-7e overexpression. Finally, miR-let-7e overexpression activated the JAK1/STAT3 signaling pathway. In conclusion, miR-let-7e may serve an important role in the progression and
\end{abstract}

Correspondence to: $\mathrm{Dr}$ Chunping Yang, Department of Otorhinolaryngology, Head and Neck Surgery, The Second Affiliated Hospital of Nanchang University, 1 Minde Road, Nanchang, Jiangxi 330006, P.R. China

E-mail: chunpingyang823@sina.com

*Contributed equally

Key words: allergic rhinitis, microRNA-let-7e, suppressor of cytokine signaling 4 , Janus kinase 1 /signal transducer and activator of transcription 3 pathway, inflammation development of AR, while overexpression of miR-let-7e had an anti-inflammatory effect by targeting SOCS4, which may be achieved by activation of the JAK1/STAT3 signaling pathway.

\section{Introduction}

Allergic rhinitis (AR), the most common form of noninfectious rhinitis, is characterized by chronic inflammation of the nasal mucosa and hypersensitivity (1). AR is a common disease that serves as a risk factor for the development of other diseases, such as asthma (2). The symptoms of AR, including nasal congestion, rhinorrhea and sneezing, considerably affect the daily activities, quality of sleep and work productivity of patients (3). Due to the effects of AR on quality of life, as well as its increasing prevalence (4), it is necessary to identify an effective diagnostic and treatment strategy for AR patients.

The mechanism underlying the occurrence of AR is complicated. Currently, it is generally considered that the emergence of AR is closely associated with the activation and infiltration of mast cells, eosinophils, basophils and CD $4^{+}$ T-helper type 2 cells, as well as the generation and release of various inflammatory mediators (5). MicroRNAs (miRNAs) are small noncoding RNAs, which bind to the 3'-untranslated region (3'-UTR) of target genes and function mainly as suppressors of gene expression at the post-transcriptional level $(6,7)$. In addition, miRNAs regulate the activity of immune cells within the innate and adaptive immune systems $(8,9)$, and their aberrant expression may result in immune disorders. Previous studies have emphasized the important role of miRNAs in controlling allergic airway inflammation $(10,11)$. Furthermore, Teng et al (12) observed that that miRNA-143 involved in the pathologic process of AR, which was significantly down-regulated in nasal mucosal tissues of AR patients compared with healthy control subjects. Recently, Suojalehto et al (1) detected the abnormally expression of miR-let-7e in the nasal mucosa tissues of AR patients; however, the underling mechanism remains unclear.

The present study aimed to investigate the expression of miR-let-7e in AR and its effects on the AR occurrence at the molecular level. In addition, the study further explored the underlying molecular mechanism of miR-let-7e involved in 
AR progression in order to provide the necessary theoretical basis for understanding the etiology of AR and a novel insight for the diagnosis and treatment of this disease.

\section{Materials and methods}

Experimental animals. A total of 20 specific pathogen-free male BALB/c mice (age, 6-7 weeks; weight, 16-18 g) were purchased from the Experimental Animal Center of The Second Affiliated Hospital of Nanchang University (Nanchang, China). All mice were maintained under standard conventional conditions, including a $12 \mathrm{~h}$ light/dark cycle, a temperature of $18-22^{\circ} \mathrm{C}$ and humidity of $50-60 \%$, with access to food and water ad libitum. All animal experiments were approved by the Animal Ethics Committee of The Second Affiliated Hospital of Nanchang University.

Preparation of the $A R$ mouse model. Initially, mice were intraperitoneally injected for primary sensitization, followed by intranasal treatment for local stimulation and secondary immunization. The mice were randomly divided into the AR and control groups ( $n=10$ per group). Mice in the AR group were intraperitoneally injected with $400 \mu \mathrm{l}$ saline containing $10 \mu \mathrm{g}$ ovalbumin (OVA) and $2 \mathrm{mg}$ aluminum hydroxide (Sigma-Aldrich; Merck KGaA, Darmstadt, Germany) on days 1 and 8 to promote drug sensitization. Between days 15 and 21, the mice were administered intranasal drops of $20 \mu \mathrm{l}$ saline containing $200 \mu \mathrm{g}$ OVA once daily to continuously challenge AR. Mice in the control group were injected intraperitoneally or treated intranasally with an identical dose of saline at the same time points. The mice were sacrificed $24 \mathrm{~h}$ following the final intranasal challenge (day 22). Nasal mucosa samples were then collected by swabbing the anterior nares from all mice.

Serological analysis. Blood samples were collected from the heart of each mouse $24 \mathrm{~h}$ following the final intranasal challenge to determine the histamine content of the serum. The blood was clotted at room temperature for $2 \mathrm{~h}$ and then centrifuged at $2,000 \mathrm{x}$ g for $20 \mathrm{~min}$ at $4^{\circ} \mathrm{C}$ to obtain the serum. Subsequently, the serum levels of histamine (ab213975; dilution 1:4,000; Abcam, Cambridge, UK), anti-OVA specific Immunoglobulin (Ig) E (3010; dilution 1:4,000; Chondrex, Inc., Redmond, WA, USA) and tumor necrosis factor- $\alpha$ (TNF- $\alpha$; MTA00B; dilution 1:4,000; R\&D Systems Inc., Minneapolis, MN, USA) in each mouse were measured using ELISA. The concentrations of histamine, anti-OVA specific $\mathrm{IgE}$ and TNF- $\alpha$ were calculated from the equations obtained from the standard curve plots drawn against the standard solutions provided in the kits.

Collection of human nasal mucosal specimens from rhinitis patients. Human nasal mucosal samples were obtained from the inferior turbinate sections of 23 patients with perennial AR and 18 patients with nonallergic rhinitis (NAR), serving as the controls. The AR patients included 13 males and 10 females with a mean age of 34.1 years (range, 20-62 years), while the NAR patients included 11 males and 7 females with a mean age of 32.3 years (range, 22-60 years). All patients were diagnosed according to the Clinical Practice
Guidelines (13), including their medical history, nasal endoscopy examination, an allergen skin-prick test and a specific IgE assay (ImmunoCAP; Thermo Fisher Scientific, Inc., Waltham, MA, USA). Partial inferior turbinectomy was conducted for relief of nasal obstruction in the AR and NAR patients. Nasal tissues were obtained from nasal polyps and inferior turbinates in patients receiving endoscopic sinus surgery. These samples were used for measuring the expression of miR-let-7e by RT-qPCR or for the isolation of primary nasal epithelial cells (NECs). No patient received topical or systemic corticosteroid therapy 4 weeks prior to participation into the present study. The study protocol was approved by the Ethics Committee of the Second Affiliated Hospital of Nanchang University, and written informed consents were signed by all patients.

Isolation and culture of primary NECs from rhinitis patients. Human primary NECs were isolated from the inferior turbinate tissues of 23 AR patients, as previously described (14). Following isolation, the cells were cultured in bronchial epithelial growth medium (Lonza Group, Ltd., Walkersville, $\mathrm{MD}, \mathrm{USA}$ ) at $37^{\circ} \mathrm{C}$ in humidified air with $5 \% \mathrm{CO}_{2}$. The NECs were passaged when a $70-80 \%$ confluent monolayer appeared, and passage 2 cells were used for subsequent experiments. In addition, 293T cells (American Type Culture Collection, Manassas, VA, USA) were cultured in Dulbecco's modified Eagle's medium (Sigma-Aldrich, Merck KGaA) supplemented with $10 \%$ fetal bovine serum (FBS; Sigma-Aldrich; Merck $\mathrm{KGaA}$ ), 100 units/ml penicillin, $100 \mathrm{mg} / \mathrm{ml}$ streptomycin (Invitrogen, Thermo Fisher Scientific, Inc.) and maintained in $5 \% \mathrm{CO}_{2}$ at $37^{\circ} \mathrm{C}$.

Lentivirus production and infection in NECs. A recombinant lentiviral expression plasmid (pLVX-IRESZsGreen+miR-let-7e) with green fluorescent protein (GFP) was constructed and confirmed by DNA sequencing. In order to generate lentiviral particles, the recombinant expression plasmid was co-transfected into $293 \mathrm{~T}$ cells with a packaging plasmid system (psPAX2 and pMD2G). The resultant viral particles were harvested after $48 \mathrm{~h}$ of transfection. Next, NECs were infected with the miR-let-7e lentiviral vector or with a negative-control vector without miR-let-7e at a multiplicity of infection of $60 \mathrm{pfu}$ per cell using $8 \mu \mathrm{g} / \mathrm{ml}$ polybrene infection reagent (Sigma-Aldrich, Merck KGaA) and were incubated for 2-3 days at $37^{\circ} \mathrm{C}$. The infection efficiency was then detected under a fluorescence microscope according to the GFP expression, which was detected by fluorescence microscopy (TE300; Nikon Corporation, Tokyo, Japan).

To examine the effect of enhanced suppressor of cytokine signaling 4 (SOCS4) expression, a SOCS4 overexpressing vector (pcDNA3.1-SOCS4) was constructed by sub-cloning the full-length wild-type (WT) SOCS4 coding sequence into pcDNA3.1 [forward (+)], which was also confirmed by sequencing. The pcDNA plasmids were designed and produced by GenePharma (Shanghai, China) and transfected into NECs according to the manufacturer's protocol. Briefly, the wild-type version was PCR-amplified and inserted between the BamHI and NotI restriction sites of the pcDNA3.1+ vector. Then cDNA was generated from the cells with the oligonucleotides UpBam and DownNot1. GFP was detected using an anti-GFP 
Table I. Primers used for target amplification.

\begin{tabular}{lll}
\hline Gene & \multicolumn{1}{c}{ Forward primer $\left(5^{\prime}-3^{\prime}\right)$} & \multicolumn{1}{c}{ Reverse primer $\left(5^{\prime}-3^{\prime}\right)$} \\
\hline SOCS4 & TGTTCGTCCATTGAGTTGGA & GAAGCACTGTTGGCAGTTAT \\
JAK1 & CTTCTCTGAAGTAGCTTTGGAAAG & AATAGTGGTGAACATCTAGGAGAG \\
STAT3 & GCTTCCTGCAAGAGTCGAAT & ATTGGCTTCTCAA GATACCTG \\
GAPDH & TGGACTCCACGACGTACTCAG & CGGGAAGCTTGTCATCAATGGAA
\end{tabular}

SOCS4, suppressor of cytokine signaling 4; JAK1, Janus kinase 1; STAT3, signal transducer and activator of transcription 3; GAPDH, glyceraldehyde 3-phosphate dehydrogenase.
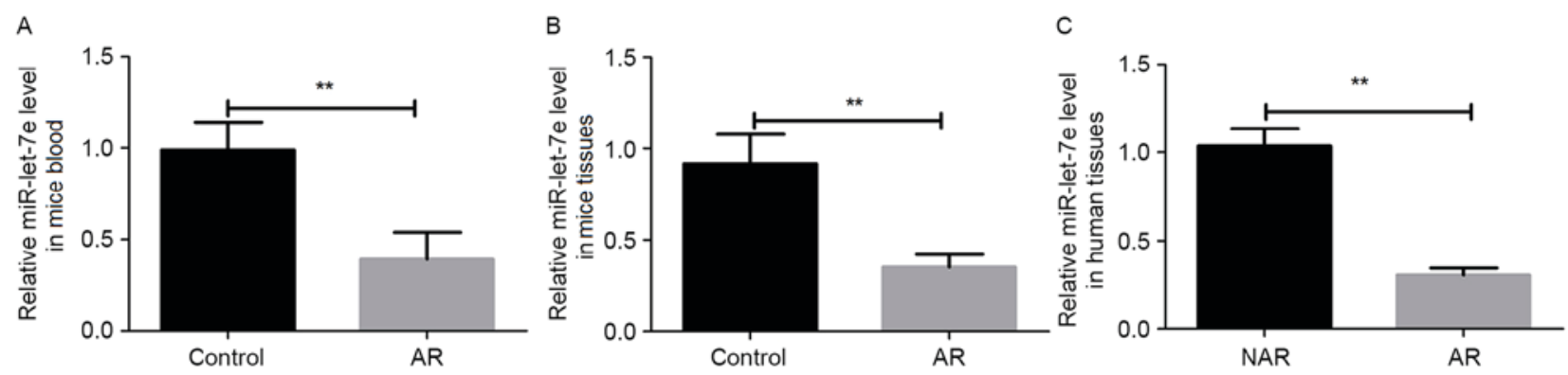

Figure 1. Relative expression levels of miR-let-7e in the (A) serum and (B) tissues of AR mice, and (C) in the tissues of patients with AR or NAR, detected by reverse transcription-quantitative polymerase chain reaction. ${ }^{* *} \mathrm{P}<0.01$ vs. the control or NAR group. AR, allergic rhinitis; NAR, nonallergic rhinitis.

rabbit IgG. Transfection with an empty construct pcDNA3.1 was performed in the control group.

Interleukin-13 (IL-13) stimulation of NECs. Following lentiviral infection, NECs were stimulated with 10 or $50 \mathrm{ng} / \mathrm{ml}$ IL-13 (R\&D Systems, Inc., Minneapolis, MN, USA) for $24 \mathrm{~h}$ or 14 days in bronchial epithelial growth medium. In the control group the NECs were cultured in bronchial epithelial growth medium supplemented with $10 \%$ FBS without test substances. The medium was changed twice a week. Cell supernatants and pellets were collected and centrifuged for $3 \mathrm{~min}$ at $100 \mathrm{x} \mathrm{g}$ at $4^{\circ} \mathrm{C}$ for reverse transcription-quantitative polymerase chain reaction (RT-qPCR) and western blot analysis to determine the mRNA and protein levels, respectively, of the target genes.

Prediction of miR-let-7e target genes. The target genes of miR-let-7e were predicted based on three public bioinformatics databases, including miRanda (www.microrna.org/) and TargetScan Human Release 6.2 (www.targetscan.org/) (15). The target genes were selected by miRanda if the mirSVR score $\leq-0.1$ and by TargetScan if the total context score was $\leq-0.1$ as previously descried (16). As predicted by miRanda and TargetScan, there was a complementary sequence between miR-let-7e and SOCS4 3'UTR therefore the levels of these were further examined.

Dual-luciferase reporter assay. The 3'-UTR of SOCS4, with WT or mutant (Mut) binding sites for miR-let-7e, was amplified and cloned into the XbaI site of the pGL3 vector (Promega Corp., Madison, WI, USA) to generate the plasmid pGL3-WT-SOCS4-3'-UTR or pGL3-Mut-SOCS4-3'-UTR, respectively. The mutant 3 '-UTR was constructed by inserting

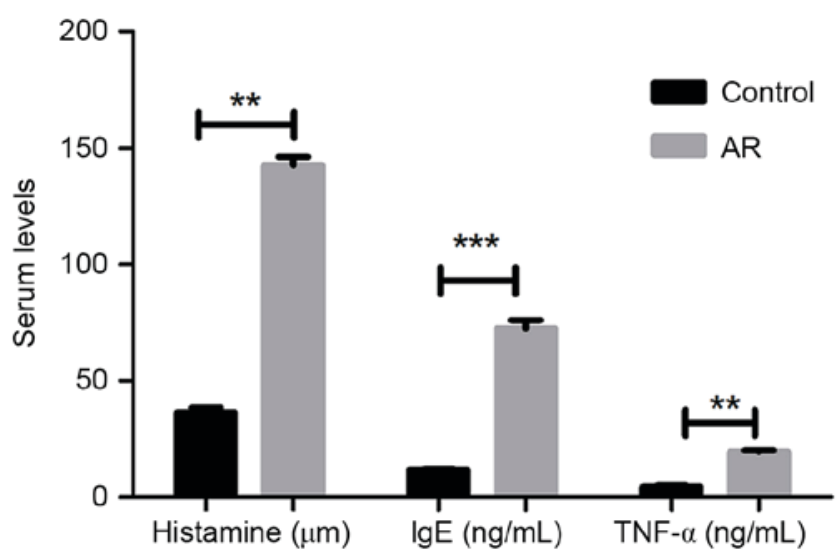

Figure 2. Expression levels of inflammatory factors histamine, IgE and TNF- $\alpha$ in the blood of AR mice, detected by enzyme-linked immunosorbent assay. ${ }^{* *} \mathrm{P}<0.01$ and ${ }^{* * *} \mathrm{P}<0.001$ vs. the control group. TNF- $\alpha$, tumor necrosis factor- $\alpha$; AR, allergic rhinitis.

five mismatch mutations into the putative seed regions of SOCS4. For the luciferase reporter assay, NECs were co-transfected with luciferase reporter vectors and with miR-let-7e mimic or the corresponding negative control (GenePharma Co., Ltd., Shanghai, China). The pRL-TK plasmid (Promega Corp.) was used as a normalizing control. The luciferase activity was analyzed using a Dual-Luciferase Reporter Assay System (Promega Corp.) after 48 h of incubation.

$R N A$ extraction and $R T-q P C R$. Total RNA was extracted from tissues isolated from mice and humans and NECs using an RNAiso Plus reagent (Takara Bio, Inc., Dalian, China). RNA 

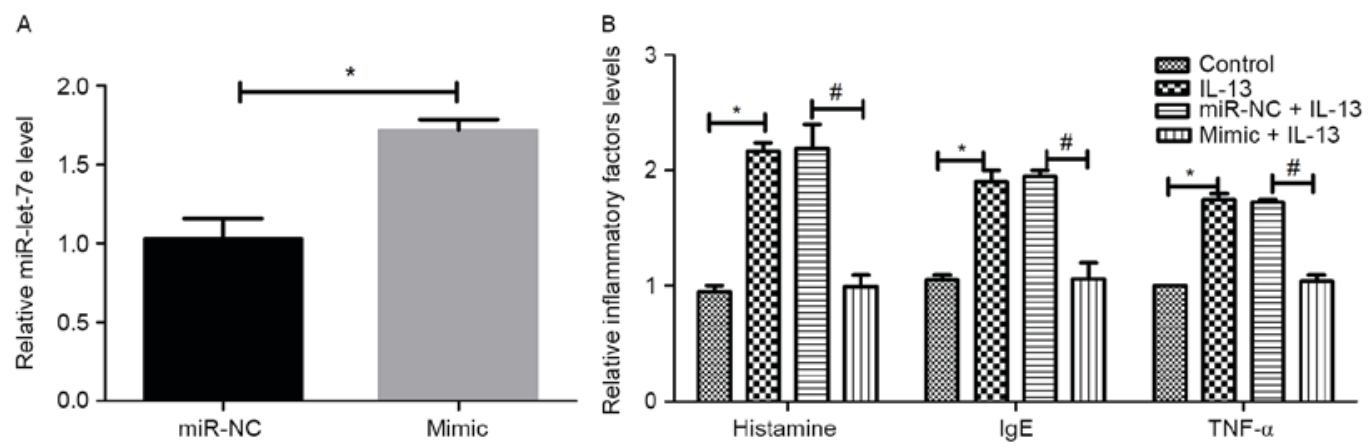

Figure 3. (A) Relative expression levels of miR-let-7e following cell transfection, detected by transcription-quantitative polymerase chain reaction (B) Expression levels of inflammatory factors histamine, IgE and TNF- $\alpha$ in IL-13-stimulated human primary nasal epithelial cells, detected by enzyme-linked immunosorbent assay. ${ }^{\mathrm{P}}<0.05$ vs. the control group; ${ }^{\prime} \mathrm{P}<0.05$ vs. the miR-NC + IL-13 group. TNF- $\alpha$, tumor necrosis factor- $\alpha$; IL, interleukin; miR, microRNA; $\mathrm{NC}$, negative control.

\section{A}

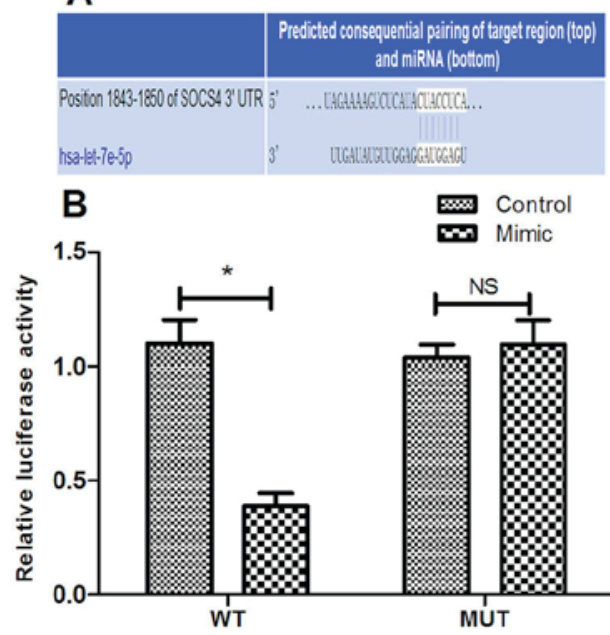

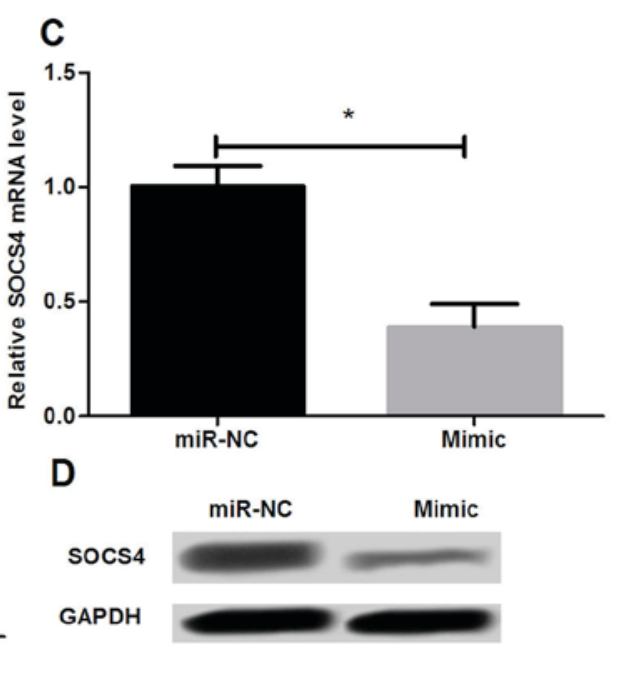

Figure 4. (A) Gene sequences of SOCS4 regulated by miR-let-7e. (B) Relative luciferase activities in WT 3'-UTR of SOCS4 and MUT SOCS4 3'-UTR in transfected cells. Relative (C) mRNA and (D) protein expression levels of SOCS4 in transfected cells, detected by reverse transcription-quantitative polymerase chain reaction and western blot analysis, respectively. ${ }^{~} \mathrm{P}<0.05$ vs. the control group. miR, microRNA; NC, negative control; WT, wild-type; MUT, mutant; UTR, untranslated region; SOCS4, suppressor of cytokine signaling 4; NS, non-significant.

concentration was assessed by a spectrophotometer (DU800, Beckman Coulter, Inc., Brea, CA, USA) at 260 and $280 \mathrm{~nm}$. RNA purity was measured by spectrophotometer (MD2000D; Civic BioScience Ltd., Beloeil, QC, Canada). Subsequent to measuring the RNA concentration and purity, total RNA was reverse transcribed into cDNA using PrimeScript RT Reagent kit (Promega Corp.). Primers were designed with Primer 5.0 software (Premier Biosoft International, Palo Alto, CA, USA) and the sequences are shown in Table I. The reverse primer used for miR-let-7e and U6 (internal control) was the Uni-miR qPCR primer, which was provided by the SYBR PrimeScript miRNA RT-PCR kit (Takara Biotechnology Co., Ltd., Dalian, China). The RT reaction and qPCR were performed with an ABI PRISM 7300 Fast Real-Time PCR system (Thermo Fisher Scientific, Inc.). PCR was performed under the following parameters: 1 predenaturation cycle of $1 \mathrm{~min}$ at $94^{\circ} \mathrm{C}, 36$ cycles of $95^{\circ} \mathrm{C}$ for $30 \mathrm{sec}, 60^{\circ} \mathrm{C}$ for $30 \mathrm{sec}, 72^{\circ} \mathrm{C}$ for $2 \mathrm{~min}$, and a final extension at $72^{\circ} \mathrm{C}$ for $5 \mathrm{~min}$. The PCR mixture $(50 \mu \mathrm{l})$ contained $10 \mathrm{ng}$ of linearized plasmid DNA, 1xAmpliTaq Gold PCR buffer (Applied Biosystems, Foster City, CA, USA), $1.5 \mathrm{mM} \mathrm{MgCl} \mathrm{M}_{2}$
(Applied Biosystems; Thermo Fisher Scientific, Inc.), $0.2 \mathrm{mM}$ deoxynucleoside triphosphates (Thermo Fisher Scientific, Inc.), $0.3 \mu \mathrm{M}$ forward primer, $0.3 \mu \mathrm{M}$ reverse primer, and $5 \mathrm{U} / \mu 1$ DyNAzyme II polymerase. The products were separated by electrophoresis on $0.2 \%$ agarose gels, and the expression levels were calculated using the $2^{-\Delta \Delta C q}$ method (17).

Western blot analysis. Total protein was extracted using radioimmunoprecipitation assay cell lysis buffer (BD Biosciences, San Jose, CA, USA) and the protein concentration was determined by a bicinchoninic acid protein assay kit (Pierce; Thermo Fisher Scientific, Inc.). Next, protein was separated by $12 \%$ SDS-PAGE and blotted on nitrocellulose membranes. The membranes were blocked with $1 \%$ TBS-Tween-20 containing $5 \%$ non-fat milk for $1 \mathrm{~h}$ at room temperature, washed with PBS three times and subsequently incubated with monoclonal antibodies at $4^{\circ} \mathrm{C}$ overnight. The primary antibodies used were as follows: Anti-SOCS4 antibodies (ab170437; dilution 1:1,000), anti-p-Janus kinase 1 (JAK1) antibodies (ab138005; dilution 1:1,000), anti-JAK1 antibodies (ab47435; dilution 

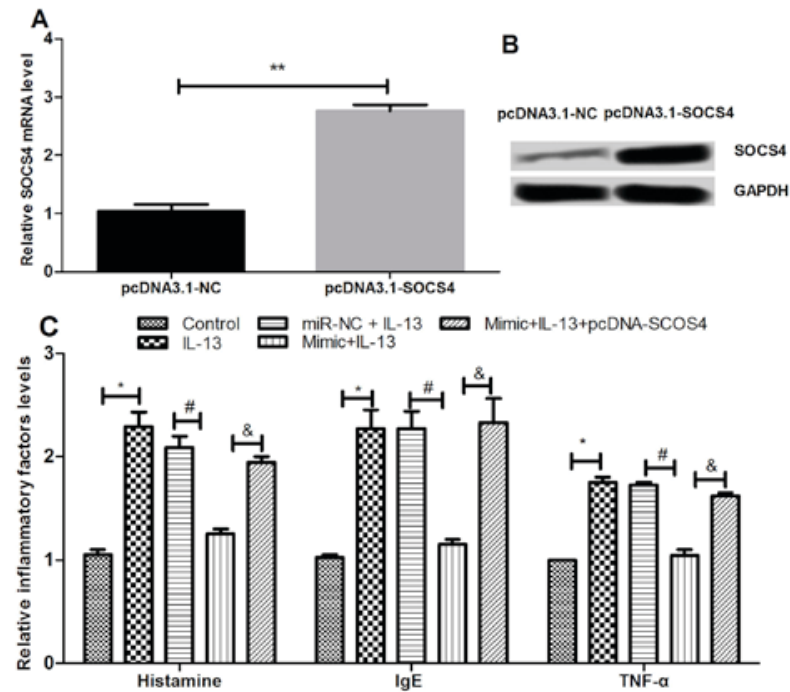

Figure 5. Relative (A) mRNA and (B) protein expression levels of SOCS4 following transfection with SOCS4 overexpressing vector or the corresponding control vector as detected by reverse transcription-quantitative polymerase chain reaction and western blot analysis, respectively. (C) Expression levels of inflammatory factors histamine, IgE and TNF- $\alpha$ in IL-13 stimulated human primary nasal epithelial cells detected by enzyme-linked immunosorbent assay. ${ }^{*} \mathrm{P}<0.05$ and ${ }^{* *} \mathrm{P}<0.01$ vs. control group; ${ }^{*} \mathrm{P}<0.05$ vs. miR-NC $+\mathrm{IL}-13$ group; ${ }^{\&} \mathrm{P}<0.05$ vs. miR-let-7e mimic + IL-13 group. SOCS4, suppressor of cytokine signaling 4 ; TNF- $\alpha$, tumor necrosis factor- $\alpha$; miR, microRNA; NC, negative control; IL, interleukin.

1:1,000), anti-p-signal transducers and activators of transcription 3 (STAT3) antibodies (ab76315; dilution 1:1,000), anti-STAT3 antibodies (ab68153; dilution 1:1,000). GAPDH (ab9485; dilution 1:2,000) was used as an internal reference. The membranes were then incubated with horseradish peroxidase-conjugated goat anti-rabbit secondary antibodies (ab6721; dilution 1:5,000) for $1 \mathrm{~h}$ at room temperature. All antibodies were purchased from Abcam. Signals were visualized with SuperSignal West Pico Chemiluminescent substrate (Pierce; Thermo Fisher Scientific, Inc.). The samples in each group were analyzed three times.

Statistical analysis. Data are expressed as the mean \pm standard error of the mean. Differences were analyzed by a two-tailed t-test or one-way analysis of variance, followed by post-hoc Bonferroni tests. Statistical analysis was conducted using SPSS software (version 16.0; SPSS, Inc., Chicago, IL, USA) for Windows. Differences with a P-value of $<0.05$ were considered as statistically significant.

\section{Results}

Expression of miR-let-7e in blood samples and tissues of mice and patients. The expression levels of miR-let-7e in the blood and nasal mucosa of AR mice were detected using RT-qPCR. The result revealed that the expression of miR-let-7e was significantly decreased in the serum and nasal mucosa tissue of AR mice when compared with the control group $(\mathrm{P}<0.01$; Fig. 1A and B). In addition, the nasal mucosa tissues of AR and NAR patients were also collected, and the expression levels of miR-let-7e in these tissues were detected. As shown in Fig. 1C, the expression of miR-let-7e was significantly lower in the nasal mucosa of AR patients in comparison with that in NAR patients $(\mathrm{P}<0.01)$.

Expression of inflammatory factors in mouse blood samples. Subsequent to successfully establishing an AR mouse model with OVA administration, the protein expression levels of inflammatory factors histamine, IgE and TNF- $\alpha$ in the mouse blood were determined by ELISA. As presented in Fig. 2, the three serum inflammatory factors presented significantly higher levels in AR mice as compared with those in the control group $(\mathrm{P}<0.01$ or $\mathrm{P}<0.001)$.

Effect of miR-let-7e overexpression on inflammatory factor expression. To investigate the association between miR-let-7e and inflammatory factors, NECs were isolated from AR patients and transfected with miR-let-7e mimic or negative control. Subsequently, NECs were stimulated with IL-13 to observe the regulatory association between miR-let-7e overexpression and inflammatory factors. The transfection efficiency is shown in Fig. 3A. Compared with the control, the expression of miR-let-7e increased significantly in the miR-let-7e mimic group $(\mathrm{P}<0.05)$, suggesting that miR-let-7e overexpression was successfully performed. As revealed in Fig. 3B, the administration of IL-13 significantly increased the expression of the inflammatory factors, histamine, IgE and TNF- $\alpha$ compared with the control group $(\mathrm{P}<0.05)$. However, no significant differences were observed between the IL-13 group and the miR-NC+IL-13 group. The effects of IL-13 were reversed by miR-let-7e overexpression in the mimic+IL-13 group compared with the miR NC+IL-13 group $(\mathrm{P}<0.05)$.

Prediction and examination of the targeting effect of miR-let-7e on SOCS4. The candidate target genes of miR-let-7e were predicted based on three public miRNA databases. It was observed that the 3'-UTR of SOCS4 was a potential miR-let-7e binding site, suggesting that SOCS4 may be a direct target of miR-let-7e in AR (Fig. 4A). Subsequently, a dual-luciferase reporter assay was conducted to further validate whether miR-let-7e is able to bind to the 3'-UTR of SOCS4 in NECs. The results demonstrated that overexpression of miR-let-7e by transfection with miR-let-7e mimic resulted in significant suppression of the SOCS4-3'-UTR reporter luciferase activity $(\mathrm{P}<0.05)$, while the mutant SOCS4-3'-UTR abrogated the suppressive effect of the miR-let-7e mimic (Fig. 4B). In addition to the luciferase activity analysis, the mRNA and protein levels of SOCS4 in NECs were detected by RT-qPCR and western blot analysis, respectively. It was identified that miR-let-7e overexpression significantly inhibited the endogenous mRNA and protein expression levels of SOCS4 $(\mathrm{P}<0.05$; Fig. 4C and D), indicating that miR-let-7e may negatively regulate SOCS4 expression in NECs.

Effects of SOCS4 overexpression on inflammatory factor expression. The study then investigated the effect of SOCS4 overexpression on the expression levels of various inflammatory factors by transfection of NECs with pcDNA3.1-SOCS4. As shown in Fig. 5A and B, SOCS4 was significantly overexpressed following the transfection $(\mathrm{P}<0.01)$. The expression levels of histamine, IgE and TNF- $\alpha$ subsequent to SOCS4 overexpression were detected using ELISA. As presented in 


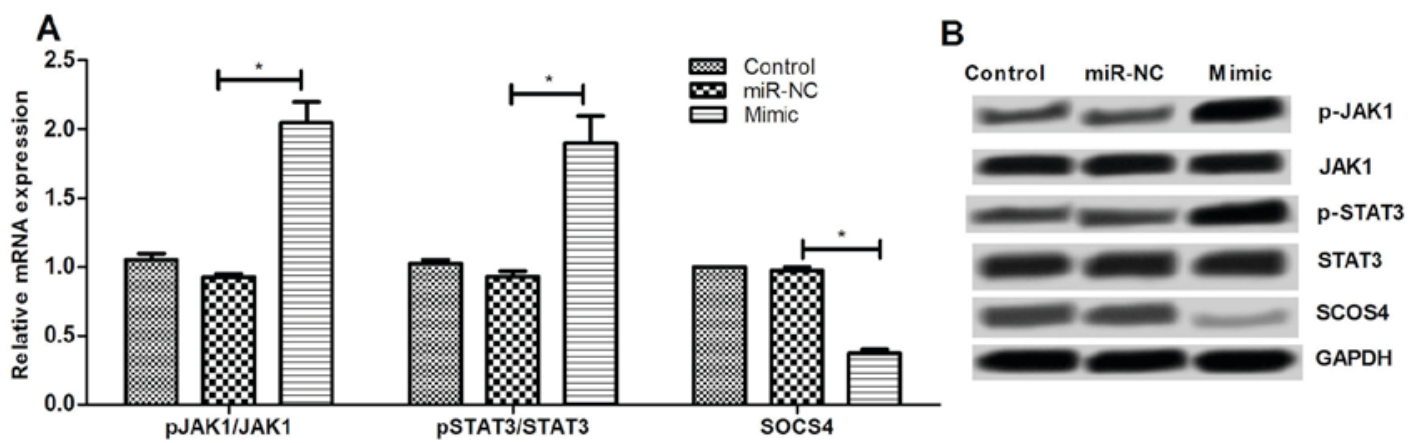

Figure 6. Expression levels of JAK1, STAT3 and SOCS4 in transfected cells, detected by (A) reverse transcription-quantitative polymerase chain reaction and (B) western blot analysis. "P<0.05 vs. miR-NC group. SOCS4, suppressor of cytokine signaling 4; miR, microRNA; NC, negative control; JAK1, Janus kinase 1; STAT3, signal transducer and activator of transcription 3.

Fig. 5C, the results revealed that the inflammatory factors were significantly increased by IL-13 compared with the control, but this effect was significantly reversed by simultaneous overexpression of miR-let-7e and IL-17. The results demonstrated that overexpressed SOCS4 abrogated the effect of miR-let-7e overexpression on the inflammatory factors induced by IL-13 $(\mathrm{P}<0.05)$. This suggested that miR-let-7e may inhibit inflammation by regulating the expression of SOCS4.

Effect of miR-let-7e overexpression on JAK1/STAT signaling pathway. To further examine the underlying mechanisms of miR-let-7e regulating SOCS4, the effect of miR-let-7e on JAK1/STAT3 pathway was determined. It was observed that miR-let-7e overexpression significantly increased the mRNA and protein expression levels of p-JAK1 and p-STAT3, as well as decreased the SOCS4 expression $(\mathrm{P}<0.01$; Fig. 6). These results suggested that miR-let-7e may regulate the SOCS4 expression via activating the JAK1/STAT3 pathway.

\section{Discussion}

The present study demonstrated that miR-let-7e was downregulated in patients and mice with AR when compared with the controls. miR-let-7e overexpression inhibited the expression levels of various inflammatory factors in AR mice and IL-13-stimulated NECs. Further experiments identified that SOCS4 was a potential target gene of miR-let-7e and was negatively regulated by miR-let-7e. In particular, overexpressed SOCS4 was able to abrogate the anti-inflammatory activity of miR-let-7e overexpression. Furthermore, it was observed that miR-let-7e overexpression activated the JAK1/STAT3 signaling pathway.

Previous studies have suggested the role of miRNAs in controlling allergic airway inflammation $(10,11)$. The miR-let-7 family of miRNAs is highly conserved across animal species, serving important roles in the regulation of cell proliferation and differentiation (18). Notably, altered expression of miR-let-7 has also been reported in inflammation. For instance, Iliopoulos et al (19) have demonstrated that miR-let-7 is repressed in inflammation, resulting in increased expression of pro-inflammatory cytokines and enhanced inflammatory responses. The results of the present study revealed the lower expression of miR-let-7 in patients with
AR and a mouse model of AR, which was consistent with a previous study (1). The results also indicated that overexpression of miR-let-7 may exert an anti-inflammatory effect on the development of AR. Kumar et al (20) administrated miR-let-7 mimics to mice and observed that overexpression of miR-let-7 repressed IL-13 production and reduced the inflammation in the allergic airway inflammation. IL-13 is a cytokine produced by $\mathrm{T}$ helper type 2 cells and it has been confirmed that IL-13 is required for allergen-induced airway inflammation and the production of mucus (21). In accordance with the aforementioned studies, the results of the current study also observed that administration of IL-13 significantly increased inflammatory factors, whereas miR-let-7e overexpression inhibited the expression levels of the inflammatory factors histamine, IgE and TNF- $\alpha$, suggesting the anti-inflammatory activity of miR-let-7e.

SOCS4 belongs to the SOCS family that is composed of eight members, including cytokine-inducible Src-homology 2 protein (CIS), as well as SOCS1 to $7(22,23)$. These proteins are known to be cytokine-inducible negative regulators of cytokine signaling, as well as key regulators of innate and adaptive immunity (24). Galic et al (25) also reported that the SOCS family proteins serve an critical role in mediating inflammatory responses in immune cells and metabolic organs. In the present study, SOCS4 was a potential target gene of miR-let-7e, and was negatively regulated by this miRNA. SOCS4 overexpression was able to abrogate the anti-inflammatory activity of miR-let-7e, which suggested that the anti-inflammatory function of miR-let-7e may be achieved by regulating SOCS4. The results demonstrated that SOCS4 overexpression may serve as an important pro-inflammatory factor in the development of AR.

The JAK1/STAT3 pathway is a conserved signaling pathway employed by diverse cytokines, growth factors, interferons and associated molecules (26). This pathway transmits information from extracellular chemical signals to the nucleus, which leads to the expression of genes involved in immunity, proliferation, apoptosis and differentiation. Disrupted JAK1/STAT3 functionality results in immune deficiency syndromes (27). It has been reported that cytokine receptors are constitutively associated with members of the JAK family of protein tyrosine kinases. In addition, the STAT family of transcription factors serves a critical role in the regulation of physiological responses to cytokine stimulation (28). JAK1/STAT3 signaling has been 
reported to be closely involved in inflammation $(29,30)$. Once activated, JAK1/STAT3 signaling can lead to the expression of inflammation-associated genes (31). Notably, Alexander (32) has identified that the SOCS proteins are key negative regulators of the JAK1/STAT3 pathway. In the present study, miR-let-7e overexpression activated the JAK1/STAT3 pathway and decreased the SOCS4 expression, which suggested that miR-let-7e may negatively regulate SOCS4 in order to activate the JAK1/STAT3 signaling pathway.

In conclusion, the present study observed that miR-let-7e may serve an important role in the progression and development of AR. Overexpression of miR-let-7e exerted an anti-inflammatory effect by targeting SOCS4 in the progression and development of AR, which may be achieved by activating the JAK1/STAT3 signaling pathway. Therefore, miR-let-7e and SOCS4 may be used as biomarkers in the diagnosis and treatment of AR.

\section{Acknowledgements}

This study was supported by a grant from the Jiangxi Provincial Natural Science Foundation (no. 20151BAB205028).

\section{Competing interests}

The authors declare that they have no competing interests.

\section{References}

1. Suojalehto H, Toskala E, Kilpeläinen M, Majuri ML, Mitts C, Lindström I, Puustinen A, Plosila T, Sipilä J, Wolff H and Alenius H: MicroRNA profiles in nasal mucosa of patients with allergic and nonallergic rhinitis and asthma. Int Forum Allergy Rhinol 3: 612-620, 2013.

2. Luo Y, Deng Y, Tao Z, Chen S, Xiao B, Ren J, Chen Z, Han J, Kong Y, Xu Y and Deng M: Regulatory effect of microRNA-135a on the Th1/Th2 imbalance in a murine model of allergic rhinitis. Exp Ther Med 8: 1105-1110, 2014.

3. Walker S, Khan-Wasti S, Fletcher M, Cullinan P, Harris J and Sheikh A: Seasonal allergic rhinitis is associated with a detrimental effect on examination performance in United Kingdom teenagers: Case-control study. J Allergy Clin Immunol 120: 381-387, 2007.

4. Nihlén U, Greiff L, Montnémery P, Löfdahl CG, Johannisson A, Persson $\mathrm{C}$ and Andersson M: Incidence and remission of self-reported allergic rhinitis symptoms in adults. Allergy 61: 1299-1304, 2006.

5. Miadonna A, Milazzo N, Gibelli S, Salmaso C, Lorini M and Tedeschi A: Nasal response to a single antigen challenge in patients with allergic rhinitis-inflammatory cell recruitment persists up to 48 h. Clin Exp Allergy 29: 941-949, 1999.

6. Guo H, Ingolia NT, Weissman JS and Bartel DP: Mammalian microRNAs predominantly act to decrease target mRNA levels. Nature 466: 835-840, 2010.

7. Ding H, Wu YL, Wang YX and Zhu FF: Characterization of the microRNA expression profile of cervical squamous cell carcinoma metastases. Asian Pac J Cancer Prev 15: 1675-1679, 2014.

8. O'Connell RM, Rao DS, Chaudhuri AA and Baltimore D: Physiological and pathological roles for microRNAs in the immune system. Nat Rev Immunol 10: 111-122, 2010.

9. Chen RF, Huang HC, Ou CY, Hsu TY, Chuang H, Chang JC, Wang L, Kuo HC and Yang KD: MicroRNA-21 expression in neonatal blood associated with antenatal immunoglobulin E production and development of allergic rhinitis. Clin Exp Allergy 40: 1482-1490, 2010.

10. Jiang X: The emerging role of microRNAs in asthma. Mol Cell Biochem 353: 35-40, 2011.
11. Shaoqing Y, Ruxin Z, Guojun L, Zhiqiang Y, Hua H, Shudong Y and Jie Z: Microarray analysis of differentially expressed microRNAs in allergic rhinitis. Am J Rhinol Allergy 25: e242-e246, 2011

12. Teng Y, Zhang R, Liu C, Zhou L, Wang H, Zhuang W, Huang Y and Hong Z: miR-143 inhibits interleukin-13-induced inflammatory cytokine and mucus production in nasal epithelial cells from allergic rhinitis patients by targeting IL13R $\alpha 1$. Biochem Biophys Res Commun 457: 58-64, 2014.

13. Bousquet J, Lund VJ, van Cauwenberge P, Bremard-Oury C, Mounedji N, Stevens MT and El-Akkad T: Implementation of guidelines for seasonal allergic rhinitis: A randomized controlled trial. Allergy 58: 733-741, 2003.

14. Shi J, Luo Q, Chen F, Chen D, Xu G and Li H: Induction of IL-6 and IL- 8 by house dust mite allergen Der $\mathrm{pl}$ in cultured human nasal epithelial cells is associated with PAR/PI3K/NFkappaB signaling. ORL J Otorhinolaryngol Relat Spec 72: 256-265, 2010.

15. Peterson SM, Thompson JA, Ufkin ML, Sathyanarayana P, Liaw L and Congdon CB: Common features of microRNA target prediction tools. Front Genet 5: 23, 2014.

16. Shi H, Chen J, Li Y, Li G, Zhong R, Du D, Meng R, Kong W and Lu M: Identification of a six microRNA signature as a novel potential prognostic biomarker in patients with head and neck squamous cell carcinoma. Oncotarget 7: 21579-21590, 2016.

17. Livak KJ and Schmittgen TD: Analysis of relative gene expression data using real-time quantitative PCR and the 2(-Delta Delta C(T)) method. Methods 25: 402-408, 2001.

18. Pasquinelli AE, Reinhart BJ, Slack F, Martindale MQ, Kuroda MI, Maller B, Hayward DC, Ball EE, Degnan B, Müller P, et al: Conservation of the sequence and temporal expression of let-7 heterochronic regulatory RNA. Nature 408: 86-89, 2000.

19. Iliopoulos D, Hirsch HA and Struhl K: An epigenetic switch involving NF-kappaB, Lin28, Let-7 MicroRNA, and IL6 links inflammation to cell Transformation. Cell 139: 693-706, 2011.

20. Kumar M, Ahmad T, Sharma A, Mabalirajan U, Kulshreshtha A, Agrawal A and Ghosh B: Let-7 microRNA-mediated regulation of IL-13 and allergic airway inflammation. J Allergy Clin Immunol 128: 1077-1085.e1-10, 2011.

21. Wills-Karp M, Luyimbazi J, Xu X, Schofield B, Neben TY, Karp CL and Donaldson DD: Interleukin-13: Central mediator of allergic asthma. Science 282: 2258-2261, 1998.

22. Hilton DJ, Richardson RT, Alexander WS, Viney EM, Willson TA, Sprigg NS, Starr R, Nicholson SE, Metcalf D and Nicola NA: Twenty proteins containing a C-terminal SOCS box form five structural classes. Proc Natl Acad Sci USA 95: 114-119, 1998.

23. Jin HJ, Shao JZ, Xiang LX, Wang H and Sun LL: Global identification and comparative analysis of SOCS genes in fish: Insights into the molecular evolution of SOCS family. Mol Immunol 45: 1258-1268, 2008.

24. Kedzierski L, Linossi EM, Kolesnik TB, Day EB, Bird NL, Kile BT, Belz GT, Metcalf D, Nicola NA, Kedzierska K and Nicholson SE: Suppressor of cytokine signaling 4 (SOCS4) protects against severe cytokine storm and enhances viral clearance during influenza infection. PLoS Pathog 10: e1004134, 2014.

25. Galic S, Sachithanandan N, Kay TW and Steinberg GR: Suppressor of cytokine signalling (SOCS) proteins as guardians of inflammatory responses critical for regulating insulin sensitivity. Biochem J 461: 177-188, 2014.

26. O'Shea JJ, Schwartz DM, Villarino AV, Gadina M, Mcinnes IB and Laurence A: The JAK-STAT pathway: Impact on human disease and therapeutic intervention. Ann Rev Med 66: 311-328, 2015.

27. Aaronson DS and Horvath CM: A road map for those who don't know JAK-STAT. Science 296: 1653-1655, 2002.

28. Krebs DL and Hilton DJ: SOCS: Physiological suppressors of cytokine signaling. J Cell Sci 113: 2813-2819, 2000.

29. Darnell JE Jr: STATs and gene regulation. Science 277: 1630-1635, 1997.

30. Kessler DS, Veals SA, Fu XY and Levy DE: Interferon-alpha regulates nuclear translocation and DNA-binding affinity of ISGF3, a multimeric transcriptional activator. Genes Dev 4: 1753-1765, 1990.

31. Kim OS, Park EJ, Joe EH and Jou I: JAK-STAT signaling mediates gangliosides-induced inflammatory responses in brain microglial cells. J Biol Chem 277: 40594-40601, 2002.

32. Alexander WS: Suppressors of cytokine signalling (SOCS) in the immune system. Nat Rev Immunol 2: 410-416, 2002. 